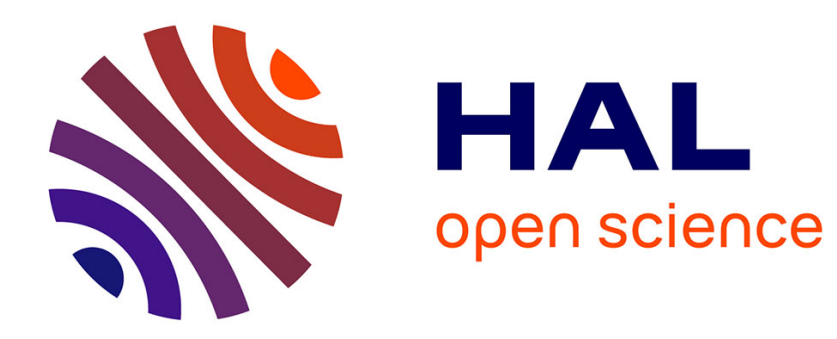

\title{
Un programme d'acquisition de données
}

\author{
J.C. Faivre, J.L. Escudié
}

\section{To cite this version:}

J.C. Faivre, J.L. Escudié. Un programme d'acquisition de données. Revue de Physique Appliquée, 1969, 4 (2), pp.131-132. 10.1051/rphysap:0196900402013100 . jpa-00243178

\section{HAL Id: jpa-00243178 https://hal.science/jpa-00243178}

Submitted on 1 Jan 1969

HAL is a multi-disciplinary open access archive for the deposit and dissemination of scientific research documents, whether they are published or not. The documents may come from teaching and research institutions in France or abroad, or from public or private research centers.
L'archive ouverte pluridisciplinaire HAL, est destinée au dépôt et à la diffusion de documents scientifiques de niveau recherche, publiés ou non, émanant des établissements d'enseignement et de recherche français ou étrangers, des laboratoires publics ou privés. 


\title{
UN PROGRAMME D'AGQUISITION DE DONNÉES
}

\author{
J. G. FAIVRE et J. L. ESGUDIÉ, \\ Service de Physique Nucléaire à Moyenne Énergie, C.E.N., Saclay.
}

\begin{abstract}
Résumé. - Nous décrivons un programme d'acquisition sur 64000 canaux pour calculateur CII 90-10. Ce programme utilise des méthodes de mémoires associatives et comprend l'identification de particules. Les contenus des canaux sont mis à jour en permanence sur mémoire de masse (disque).
\end{abstract}

Abstract. - A 64,000 channel acquisition programme has been written for the CII 90-10 (SDS 92) computer. This programme uses the associative memory methods and includes particle identification. The counts per channel are permanently stored in the mass memory (disk).

Ces lignes décrivent un système de programmes pour l'acquisition de données par calculateur en ligne, utilisant des techniques de mémoires associatives et fournissant l'information intégrée sur mémoire de masse.

I. Voulant avoir le contrôle immédiat de l'expérience, nous avons abandonné l'idée de méthodes d'analyse multiparamétrique, tandis que les méthodes d'acquisition dans des mémoires adressables (appartenant à un calculateur ou à un analyseur multicanaux) ont dû être abandonnées dans les cas où le nombre de « descripteurs » requis pour décrire une expérience devient trop grand. Disposant d'un calculateur CII C $90-10$ de $16 \mathrm{k}$ mémoires de 12 bits, nous nous sommes orientés vers un traitement en ligne utilisant des méthodes de mémoires associatives.

II. Lorsque la chaîne électronique a reconnu et digitalisé un événement, le programme "Separ », appelé sur le plus haut niveau d'interruptions prioritaires, forme les deux descripteurs d'un événement : le premier, qui indique le spectre, contient trois informations : le numéro de l'angle de détection, l'état de polarisation du faisceau, le numéro de type de particule. Gette dernière information est issue de la formation des spectres « de masse » obtenus à partir des informations « énergie » et « perte d'énergie ». Le second descripteur est l'énergie. Lorsque le nombre de descripteurs possibles est inférieur à $12 \mathrm{k}$, nous utilisons pour le rangement une méthode classique de mémoires adressables avec un rangement en « pile » des dépassements de capacité.

III. 1. Si le nombre de descripteurs possibles est supérieur à $12 \mathrm{k}$, « Separ » appelle le programme de stockage « Stocel » fonctionnant sur un mode de mémoires associatives.

III .2. La zone de mémoire disponible (environ $12 \mathrm{k}$ ) est découpée en «cellules » numérotées formées de quatre mots ( fig. 1) : $D(\mathrm{~N})$, énergie de l'événement placé dans la cellule $\mathrm{N} ; C(\mathrm{~N})$, nombre d'événements

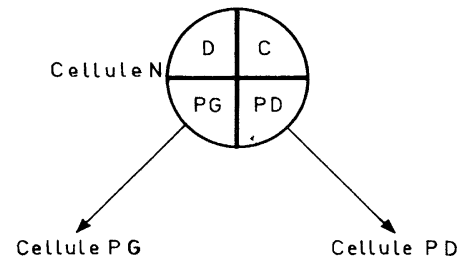

FIG. 1.

d'énergie $D(\mathrm{~N}) ; P D(\mathrm{~N})$, pointeur droit; $P G(\mathrm{~N})$, pointeur gauche.

Les pointeurs contiennent les numéros des deux cellules reliées à la cellule $\mathrm{N}$. Une valeur supérieure au nombre maximal de cellules indique l'absence de liaison. On voit que l'on peut de la sorte structurer la mémoire sans déplacer réellement les informations. Plusieurs structures utilisant ce type de cellule sont formées en mémoire.

III.3. Chaine des Gellules libres. - Un mot, lib, contient le numéro de la première cellule libre. Dans le pointeur droit de celle-ci se trouve le numéro de la seconde cellule libre, etc. Cette façon de structurer la zone de mémoire disponible est inspirée des langages de listes [1]. On voit qu'il est très facile de retirer ou de replacer une cellule dans cette chaîne, qui contient à chaque instant toutes les cellules disponibles. 
III.4. Arbre (fig. 2). - A chaque spectre est associée une structure appelée « arbre » [2]. Un mot entr(I), analogue à lib, contient le numéro de la première cellule de l'arbre (I). Celle-ci contient l'éner-

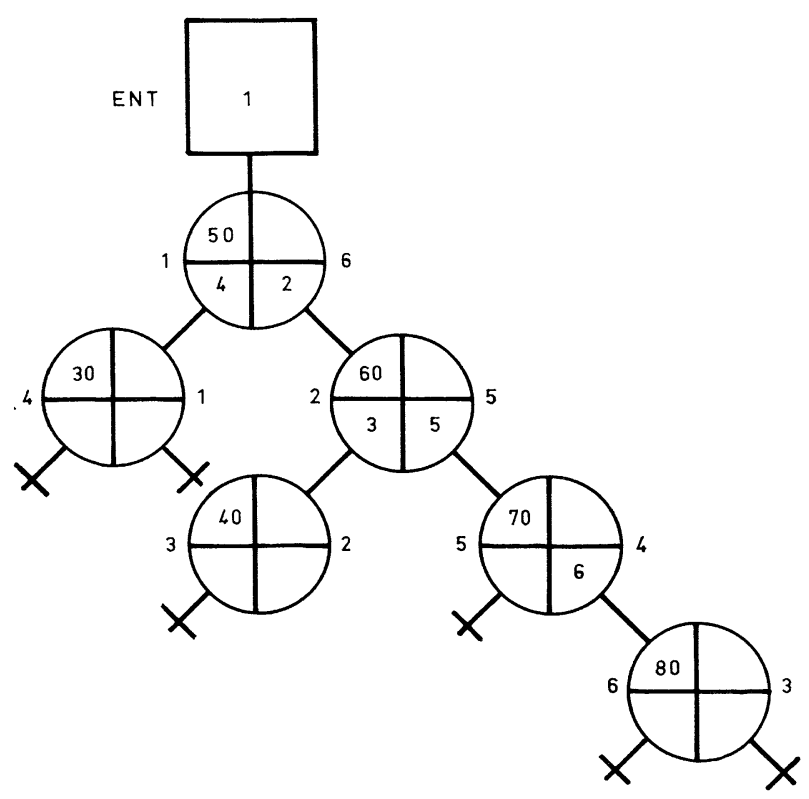

FIG. 2.

gie du premier événement arrivé dans le spectre $I$. Lorsqu'un nouvel événement se présente, on compare dans Stocel son énergie $E$ à celle qui se trouve dans la première cellule $D(1)$.

Si $E=D(1)$, on ajoute un au nombre de coups $C(1)$ de la première cellule. Si $E>D(1)$, on se dirige vers la cellule $P D(1)$. Si $E<D(1)$, on se dirige vers la cellule $P G(1)$.

Dans les deux cas, on peut avoir :

III.4.1. Absence de liaison : Ceci signifie que l'événement ne se trouve pas encore dans l'arbre. On prend alors une cellule dans la chaîne des libres et on l'ajoute à l'arbre, en y inscrivant l'énergie « $E$ » et « 1 » en contenu.

III .4 2. Liaison vers une cellule $x$ : On répète l'opération de comparaison avec $D(x)$. On peut ainsi retrouver rapidement un événement déjà stocké dans l'arbre. L'apparition d'un nouvel événement ne nécessite pas une profonde modification de la structure, mais l'accro- chage d'une simple cellule, c'est-à-dire la modification d'un seul pointeur.

III .4.3. Le programme de fond partage son temps entre la «visualisation » et la «mise à jour », qui permet de libérer la zone de stockage. La mise à jour de l'information intégrée sur mémoire de masse est faite spectre par spectre. La mémoire de masse utilisée était récemment une paire de bandes magnétiques; c'est depuis peu un disque magnétique. On effectue la succession des opérations suivantes : i) lecture sur la mémoire de masse du spectre le plus « urgent » (l'urgence étant déterminée à la fois par le remplissage du spectre et l'ancienneté de sa dernière mise à jour), et transfert de l'information en mémoire rapide dans un « bloc tampon »; ii) débranchement de l'arbre NS de son point d'entrée entr(NS) et accrochage du point d'entrée tête, pour exploitation par le programme TRSP de l'arbre ainsi figé. On se dirige systématiquement à gauche chaque fois que la liaison existe, sinon à droite. Si cette dernière liaison est également absente, la cellule est alors une « terminaison » (cellule 4 , fig. 2). Cette cellule peut être détachée sans perturber la structure; on ajoute son contenu dans le «bloc tampon », on coupe la liaison qui la relie à la cellule précédente, on raccroche cette cellule à la chaîne des libres, et l'on continue l'exploration jusqu'à ce que la première cellule de l'arbre ait été détachée; iii) écriture du bloc tampon ainsi complété sur mémoire de masse. On passe ensuite au spectre suivant dans l'ordre des urgences. Sur la figure 2, on a supposé que les cellules étaient initialement ordonnées dans la chaîne des cellules libres. Les numéros de gauche indiquent l'ordre d'arrivée des cellules dans l'arbre, alors que ceux de droite indiquent leur ordre de décrochage par TRSP.

III.4.4. Stocel ne rejette aucun des événements acceptés par Separ et transforme le descripteur d'énergie afin de rendre aléatoire la répartition des grands contenus dans les canaux, ce qui permet de se rapprocher d'une forme symétrique des arbres, pour minimiser le temps de recherche d'un descripteur. Grâce à l'existence de la chaîne des cellules libres, chaque cellule peut être affectée à n'importe quel spectre ou à la chaîne des dépassements de capacité.

Il y a deux limitations de taux de comptage : le temps de classement d'un événement et le nombre de cellules libres, qui dépend lui-même de trois facteurs : la distribution des fréquences des descripteurs, le nombre total de cellules disponibles et la vitesse d'accès à la mémoire de masse.

\section{BIBLIOGRAPHIE}

[1] Broudin, Rapport C.E.A. R-3040.

[2] Hooton (I. N.), EANDC, Conf. Karlsruhe, 1964, et références incluses. 